\title{
NA61/SHINE and EMPHATIC hadron production measurements for neutrino physics
}

\author{
Matej Pavin* \\ TRIUMF \\ E-mail: mpavinetriumf.ca
}

\begin{abstract}
Accelerator-based neutrino experiments produce neutrino parent particles, mostly pions, and kaons in proton interactions with a nuclear target. Consequently, these hadrons decay to neutrinos and give rise to a high-intensity neutrino beam. Monte Carlo models are used to estimate the hadron production in the target and the elements of the beamline. The model predictions vary significantly, and as a result, corresponding neutrino flux uncertainty is limiting the precision of many neutrino measurements. Examples of these measurements include neutrino-nucleus cross-section measurements and sterile neutrino searches. For this reason, model predictions are re-weighted with external hadron-production data. Currently, the NA61/SHINE experiment at CERN SPS performs such measurements for accelerator-based neutrino experiments. Following the success of NA61/SHINE, a complementary experiment EMPHATIC at Fermilab Test Beam Facility is being carried out to extend the data coverage to low beam momentum region not accessible to NA61/SHINE. The aim of this work is to present latest results from NA61/SHINE alongside with the prospects of the EMPHATIC experiment.
\end{abstract}

Neutrino Oscillation Workshop (NOW2018)

9 - 16 September, 2018

Rosa Marina (Ostuni, Brindisi, Italy)

${ }^{*}$ Speaker for the NA61/SHINE and EMPHATIC collaborations. 


\section{Introduction}

With the increase of detector fiducial volumes in the next generation of neutrino experiments, measurements will be hampered by systematic uncertainties. One of the limitations comes from the knowledge of neutrino flux. Accelerator-based neutrino experiments use proton beams to produce pions and kaons which decay to neutrinos. The flux prediction is limited by the accuracy of Monte Carlo hadron production models. Significant progress has been made to perform hadron production measurements for both Japanese and US accelerator-based neutrino experiments. This pioneering work is being done by NA61/SHINE experiment [1] at CERN SPS. There are two types of hadron production measurements: measurements with a thin target and with a replica target. Thin target measurements use thin nuclear target $\left(4 \% \lambda_{I}\right)$ to measure differential cross section and constrain a single type of interaction. However, in neutrino experiments, secondary hadrons can re-interact and produce tertiary hadrons which cannot be constrained with the same measurement. To take all re-interactions in the target into account, it is possible to measure hadron emission from a replica of the target used in the neutrino experiment. As a result, a specific replica target measurement is useful only for a particular neutrino experiment. Latest NA61/SHINE replica target measurements for $\mathrm{T} 2 \mathrm{~K}$ [2], a long baseline neutrino experiment in Japan are presented in the next section.

\section{NA61/SHINE experiment}

NA61/SHINE or SPS Heavy Ion and Neutrino Experiment is a hadron spectrometer at CERN SPS. Secondary $\mathrm{H} 2$ beamline at SPS supplies the experiment with hadron and ion beams between $13 \mathrm{AGeV} / \mathrm{c}$ and $160 \mathrm{AGeV} / \mathrm{c}$. The setup evolved over the years and the one presented here was used to take hadron production data for T2K. It consists of four large TPC chambers, two of which are located in the magnetic field and a small gap TPC covering the forward region. Particle identification is done by energy loss measurement $(d E / d x)$ and time-of-flight (tof) measurement by the forward time of flight wall (F-TOF). The complete setup is presented in Fig. 1. NA61/SHINE took hadron

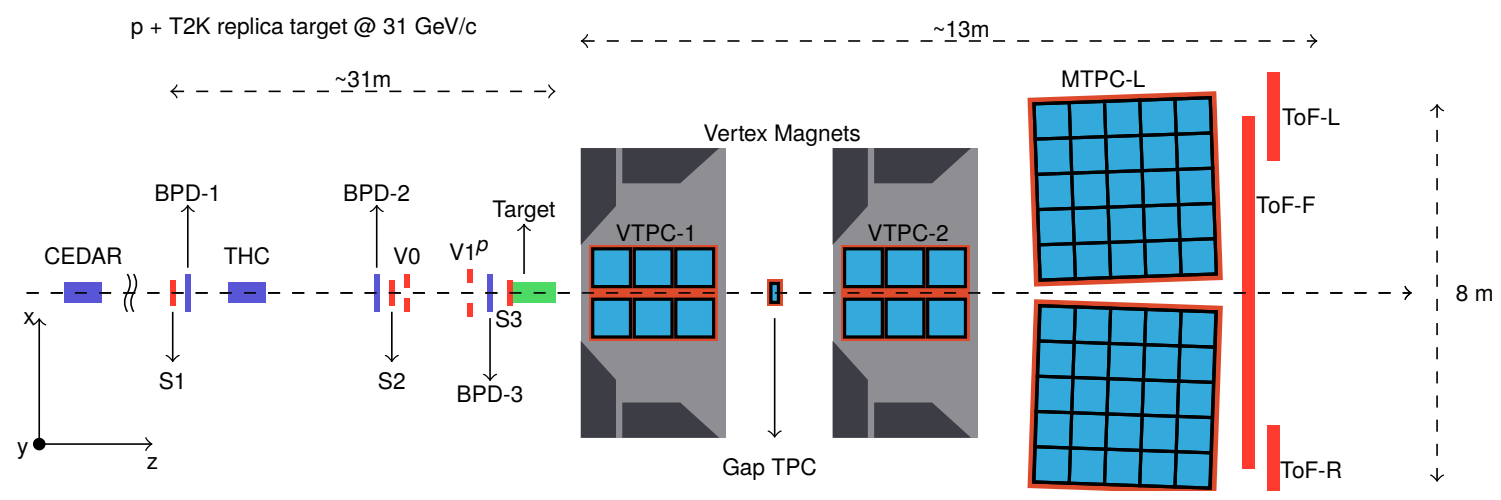

Figure 1: A top view of the NA61/SHINE experimental setup used in 2010 for the T2K replica target datataking. The beam comes from the left. The orientation of the coordinate system is shown in the bottom left corner, while its origin is located at the centre of VTPC-2.

production data for T2K between 2007 and 2010 with both the thin and the replica target. The 
beam momentum was set to $31 \mathrm{GeV} / \mathrm{c}$. The analysis of these data has been finalized, and the latest measurements with the T2K replica target. The analysis of the Fermilab hadron production data is still ongoing with recently published pion and kaon production cross-section measurements [6].

\subsection{T2K replica target measurements}

The latest $\mathrm{T} 2 \mathrm{~K}$ replica target dataset was taken in 2010 with around 10.2 million protons on target (POT). The T2K replica target is a $90 \mathrm{~cm}$ graphite rod, with a diameter equal to $2.6 \mathrm{~cm}$. The size and the target material are identical to the real T2K target without cooling. In this measurement, TPC tracks are extrapolated to the target surface and binned in momentum $(p)$, polar angle $(\theta)$ and longitudinal position of the exit point on the target surface $(z)$. Longitudinal binning is essential since hadrons emitted from different parts of the target have a different path length through the focusing magnetic field in T2K. Hadrons were identified by using tof and $d E / d x$ measurements. The raw yields were corrected for detector inefficiencies and migration effects with FLUKA 2011.2c.4 + Geant 3 simulation of the detector. Finally, results were normalized by momentum and angle bin sizes and the total POT. The results include yields of charged pions $\left(\pi^{ \pm}\right)$, charged kaons $\left(K^{ \pm}\right)$and protons [3] with a small fraction of results presented in Fig. 2. A prediction of NuBeam physics list from Geant4.10.03 is overlaid on top, and the different colors show different longitudinal regions. Systematic uncertainty is similar in size to statistical uncertainty $(<5 \%)$ for most of the bins. Due to low statistics in previous $\mathrm{T} 2 \mathrm{~K}$ replica target datasets, kaon and proton yields have been measured for the first time. Expected impact of this results is a reduction of T2K neutrino flux uncertainty to the level of $4-5 \%$. Current uncertainty was obtained after constraining flux prediction with thin target data, and it is around 10\% (see [4]). The uncertainty of low energy tail in the T2K neutrino spectra is dominated by low momentum interactions $(<10 \mathrm{GeV} / \mathrm{c})$ outside of the target. Also, CP violation measurement in atmospheric neutrinos is limited by hadron interactions in the same region. Additional measurements are needed to reduce these uncertainties.

\section{EMPHATIC}

Experiment to Measure the Production of Hadrons At a Testbeam In Chicagoland (EMPHATIC) is a new tabletop experiment ( $2 \mathrm{~m}$ in size) at Fermilab Test Beam Facility. It is complementary to NA61/SHINE, and it will take hadron production data with various targets at beam momenta mostly below $15 \mathrm{GeV} / \mathrm{c}$. First physics run is expected in late 2019. The detector will use silicon strip detectors (SiSD) with a compact permanent magnet for momentum measurement. Particle identification will be done with aerogel ring imaging Cherenkov detector (ARICH) and resistive plate chambers (RPC). First beam test with only SiSDs and various targets was done in January 2018. Collected data can be used to extract total cross-section by using optical theorem, similar to measurement in [5]. The result can be used to re-weight interaction probability in neutrino experiments. Analysis of proton-carbon forward scattering at 20,30, and $120 \mathrm{GeV} / \mathrm{c}$ is ongoing. Preliminary uncorrected differential cross-sections are shown in Fig. 3 suggest the existence of an elastic and quasi-elastic regime (see [5]) and the cross-section does not change much with incoming beam momentum. 

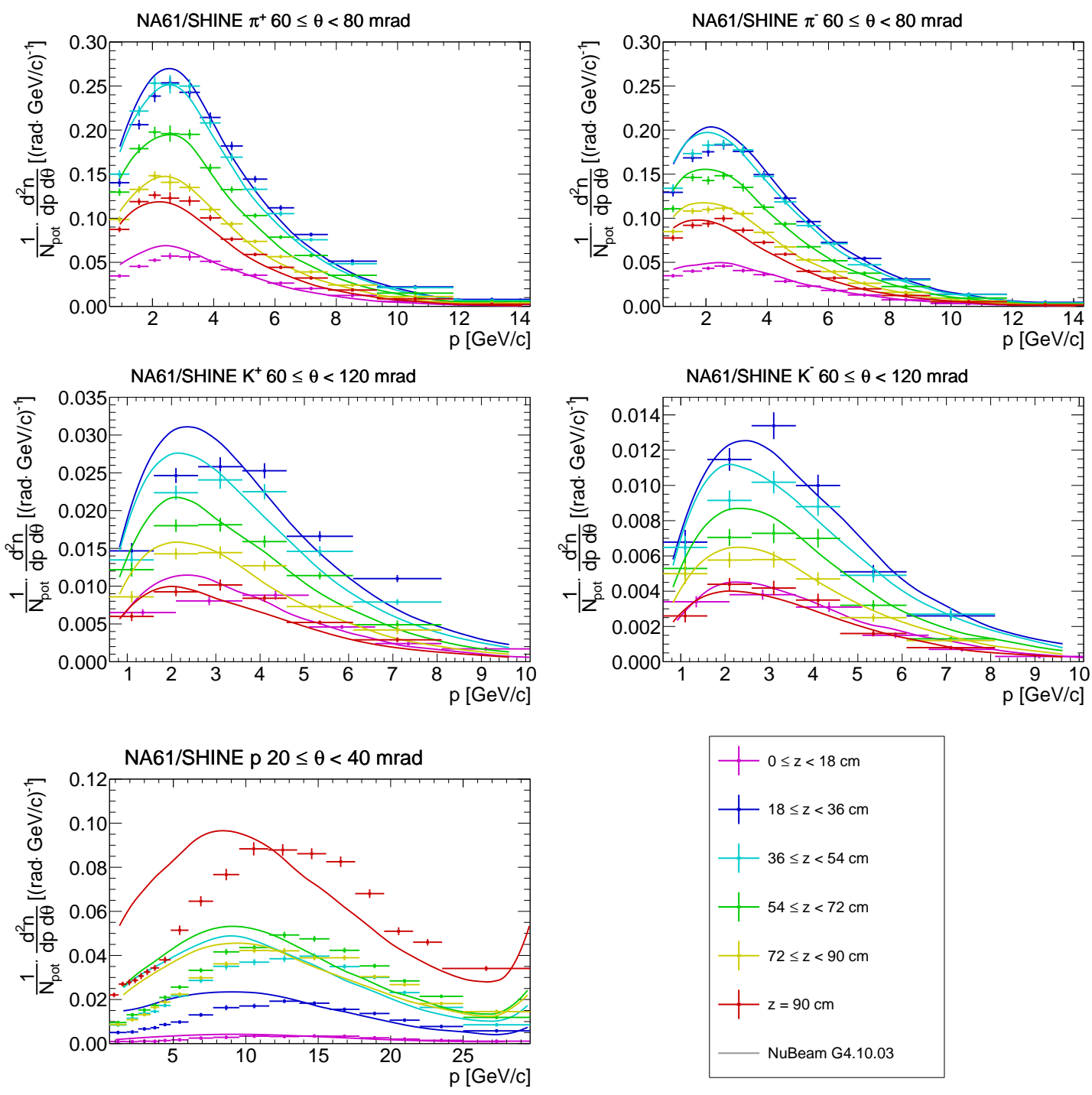

Figure 2: Double differential yields of pions, kaons, and protons emitted from the T2K replica target surface normalized by the number of protons on target. Each panel shows a narrow angular range printed on top. Different colors represent different longitudinal bins and the solid lines show NuBeam physics list from Geant4.10.03. Full angular coverage is presented in [3].

\section{Conclusion}

Hadron production measurements are crucial for reducing the uncertainty of the neutrino flux in accelerator-based neutrino experiments. NA61/SHINE released final replica target results for $\mathrm{T} 2 \mathrm{~K}$ which include pion, kaon and proton yields. The results will reduce the T2K flux uncertainty to a level of $5 \%$. However, hadron production measurements remain sparse and more data is needed to reduce the flux uncertainty in future experiments which will be limited by systematics. For this purpose, EMPHATIC is being built at Fermilab Test Beam Facility, and it will start first physics run in late 2019. 


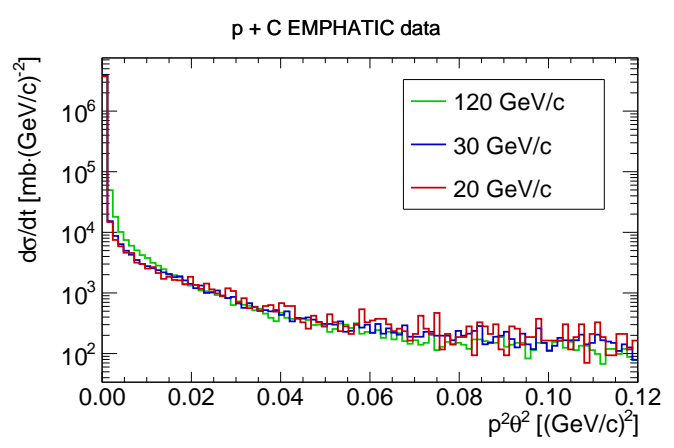

Figure 3: Uncorrected raw differential proton-carbon cross-section vs $p^{2} \theta^{2}$ for $20 \mathrm{GeV} / \mathrm{c}, 30 \mathrm{GeV} / \mathrm{c}$, and $120 \mathrm{GeV} / \mathrm{c}$ proton beams. All of the distributions overlap and differences for $p^{2} \theta^{2}<0.02(\mathrm{GeV} / \mathrm{c})^{2}$ are coming from differences in migration effects due to angular resolution and multiple scattering.

\section{References}

[1] N. Abgrall et al. [NA61 Collaboration], JINST 9 (2014) P06005

[2] K. Abe et al. [T2K Collaboration], Nucl. Instrum. Meth. A 659 (2011) 106

[3] N. Abgrall et al. [NA61/SHINE Collaboration], arXiv:1808.04927 [hep-ex].

[4] T. Vladisavljevic, arXiv:1804.00272 [physics.ins-det].

[5] G. Bellettini et al. Nucl. Phys. 79 (1966) 609.

[6] A. Aduszkiewicz et al. [NA61/SHINE Collaboration], Phys. Rev. D 98 (2018) no.5, 052001 